\title{
El Seminario de Arica
}

Entre el 23 de enero y el 2 de febrero recién pasados se reunió en la ciuclad de Arica un Seminario coauspiciado por el Centre d'Études de Politique Étrangère de París y el Instituto de Estudios Inteŕnacionales cle la Universidad de Chile, para examinar la experiencia europea y el proceso latinoamericano de integración económica y política. El Seminario contó también con el elevado auspicio del Institut de Science Economique Appliquéc, Paris; clel Istituto Affari Internazionali, Roma; del Otlo Suhr Institut, Berlín; del Institut des Hautes Études de l'Amérique Latine, París; del Forschungsinslitut der Deutschen Gesellschaft für Auswarlige Politik, Bonn; del Instiluto para la Integración de América latina, Buenos Aires; clel Instituto Brasileño de Relaciones Internacionales, Río de Janeiro; del Instituto de Estudios Peruanos, Lima y del Instituto Pcruano de Estudios del Desarrollo.

Colaboraron asimismo, con importante ayucla material y organizativa, la Junta de Adelanto de Arica, el Banco Interamericano de. Desarrollo y el Gobierno cle Cihile.

El trabajo del Seminario se organizó alrededor de la considcración de siete temas generales, cada uno examinado desde los puntos de vista europeo y latinoamericano. Se comisionó por consiguiente a catorce especialistas para que prepararan ensayos preliminares que serían presentados y discutidos en cada una de las reuniones. Cacla presentación estuvo a su vez seguida cle un primer comentalio que abrió la cliscusión detallacla del trabajo.

El programa del Seminario incluyó los siguientes temas y autores:

Développement économique et intégration: l'expérience européenne, Prof. Francois Perroux, Directeur de l'Institut di développement économique et social, París.

Integración y desarrollo: el proceso latinoamericano, Sr. Germánico Salgado, Director de Asuntos Económicos de la Organización de los Estados Americanos, Washington; comentario del Prof. Osvaldo Sun$\mathrm{kel}$, Instituto de Estudios Internacionales, Universidad de Chile.

L'agriculture dans la intégration européenne, Prof. Michel Cépède, Institut National Agronomique, París; comentario del Sr. Windfrid von Urff, Institut d'Agriculture, Université de Frankfurt.

La Agricultura en la integración latinoamericana, Sr. Jacobo Schatan, Director de la División Agrícola Conjunta, CEPAL-FAO, Santiago; 
comentario del Sr. Antonio García, Instituto de Capacitación e investigación en Reforma Agraria (ICIRA), Santiago.

L'avance technologique et scientifique et l'intégration européenne, Prof. Pierre Monbeig, Director de l'Institut dos Hautes Etudes de l'Amérique Latine, París.

El avance tecnológico y científico y la integración latinoamericana. Prof. Enrique d'Etigny, Decano de la Facultad de Ciencias Físicas y Matemáticas de la Universidad de Chile; comentario del Dr: Jorge Sábato, Director del Depto. de Metalurgia de la Comisión Nacional de Energía Atómica, Buenos Aires, y Sr. Michel Arnaudon, Secretario Ceneral del Instilut d'études du développement économique et social, París.

Problèmes financiers et monélaires de l'intégration européenne, Prof. Paul Streeten, Fellow of the Institute of Development Studies, University of Sussex (en la ausencia del autor, este trabajo fue presentado por el Sr. Andrés Bianchi, Centro Interamericano de Enseñanza de Estadistica (CIENES), Santiago); comentario del Sr. Leonhard Gleske, Presidente del Landeszentralbank, Bremen.

Problemas financicros y inonetarios de la integración latinoamericana, Sr. Carlos Massad, Presidente del Banco Central de Chile y Sr. Camilo Carrasco, Banco Central de Chile (en la ausencia de los autores este trabajo fue presentado por el Sr. Flavio Levine, Banco Central de Chile); comentario clel Prof. Enrique Iglesias, Presidente del Banco Central de Uruguay.

Les institutions de l'inlégration européenne, Sr. Altiero Spinelli, Directeur del Istitulo Affari Internazionali, Roma.

Las instituciones de la integración latinoamericana, Sr. Felipe Herrera, Presidente del. Banco Interamericano de Desarrollo (en la ausencia clel autor este trabajo fue presentado por el Sr. Eduardo Figueroa, Banco Interamericano cle Desarrollo, Washington); comentario del Sr. Torcuato di Tella, Centro Latinoamcricano de Pesquisas Sociales, Río de Janeiro.

Les Etats-Unis ct l'intégration européenne, Sr. Jacques Vernant, Sécretaire-Général du Contre d'Études de Politique Etrangère, París.

Los Estados Unidos y la integración latinoamericana, Sr. Rogelio Frigerio, ex Secretario de Relaciones Sociales de la Presidencia deArgentina; comentario del profesor Helio Jaguaribe.

La stratégie politique de l'intégration: le probleme européene, Prof. Gilbert Ziebura, Directeur de l'Otto Suhr Institut, Berlin; comentario del Sr. Alain Joxe, Instituto de Estudios Internacionales, Universidad de Chile. 
La estrategia politica de la integración: el problema latinoamericano Sr. Gustavo Lagos, Director del Instituto para la Integración de América latina, Buenos Aires.

Además participaron como presiclentes de las sesiones, asesores del director; comentaristas especializados y miembros del Comité de Estuclios, las siguientes personas:

Sr. Guillermo Anguita, Secretaría Ejecutiva para los Asuntos de la ALALC, Santiago.

Sr. Carlos F. Díaz Alejandro, Oficina de Planificación de la Presidencia de la República (ODEplan), Santiago.

Sr. Jorge Bravo Bresani, Decano de Ciencias Sociales de la Universiclacl Agraria, Lima.

Sr. Ives Gibert, Director de la Oficina de Información de las Comunidades Europeas para América latina, Montevideo.

Sr. Richard Gott, Instituto de Estudios Internacionales, Universiclad de Chile.

Prof. Amilcar Herrera, Facultad cle Giencias Físicas y Matemáticas de la Universidad de Chile.

Sr: Marcos Kaplan, Instituto de Estudios Internacionales, Universiclad de Chile.

Sr. José Matos Mar, Director del Instituto de Estudios Peruanos, Lima.

Sr. Cleantho de Paiva Leite, Instituto Brasileño de Relaciones Internacionales, Río de Janeiro.

Sr. Alberto Sepúlveda; Instituto Nacional de Desarrollo Agropecuario (INDAP), Santiago.

Sr. Luciano Tomassini, Banco Interamericano de Desarrollo, Washington.

Sr. Hubert Wicland, Jefe del Depto. cle Estudios Políticos, Ministerio de Relaciones Exteriores, Perú.

Sr. Carlos Zuranaga Flore, Presidente del Instituto Peruano de Estuclios del Desarrollo, Tima.

Las discusiones del Seminario se realizaron a un nivel sumamente satisfactorio que indudablemente constituyó un aporte valioso para los autores" de los ensayos preliminares que ahora revisarán y ampliarán sus trabajos a la lu\% cle estas discusiones para ser inmediatamente editádos en un volumen bajo lai dirección del profesor Osvaldo Sunkel. Se espera de este modo que las cleliberaciones del Seminario -que se efectuaron en privado- se encuentren reflejadas en estos ensayos y en lat introducción del profesor Sunkel que constituirán en cierto modo, las únicas conclusiones del Seminario. 
Sólo un incidente sin importancia demasiado grande empañó las tareas académicas del torneo. El Sr. Rogelio Frigerio, de Argentinit, presentó un ensayo titulado Los Eslados Unidos y la integración de América latina, que, de acuerdo con algunos de los participantes que lo comentaron, no se refirió al tema en lorma aclecuada ni fue preparado con el rigor académico que se exige en estos casos. Desgraciadamente el Sr. Frigerio interpretó estas objeciones como ataque's personales y se retiró de la sala de sesiones protestando que el Seminario era en realiclad un acto político.

Más adelante el mismo Sr. Frigerio hizo una serie de publicaciones en un perióclico tabioicle de Buenos Aires en los que calificaba en términos inconvenientes lals actividades y el auspicio de quienes parti(iparon en el Seminario. El Instituto de Estuclios Internacionales de la Universidial de chile limentó sinceramente que este incidente interrumpiera aun cuanclo nomentáneamente el clesarrollo normal y satisfactorio de los trabajos académicos del Seminario. En cuanto a las acusaciones verticlas en lia prensa tabloide de Buenos Aires, estima que éstas son singularmente inapropiadas.

Como se cxplicó mís arriba, el Seminario de Arica fue coauspiciado por el Instituto de Estudios Internacionales y. el Contre d'Éludes de Politique Élrangère en virtud de un convenio suscrito en $1960 \mathrm{y}$ debidamente ratilicado por el Hon. Consejo Universitario mediante el cual ambos centros de estudio sc comprometían a organizar un Seminario internacional cacla dos años, alternativamente en Chile y Francia. Estas reuniones tienen por objeto examinar en profundidad algunos temas principales de las relaciones internacionales políticas y cconómicas que interesen tanto a europeos como a latinoamericanos. Se alprovechó entonces, la presencia en Arica cle un grupo distinguido cle acalémicos franceses para acorclar el tema y fecha del próximo Seminario. Este sc reunirí en París, en abril de 1970, para examinar en profuncliclad el tema general, El factor militar en la politica internacional.

Aun cuando el trabajo acaclémico de las cliversas sesiones alcanzó un muy elevado nivel, el Seminario no hubiera sido tan exitoso de no haber mediado la colatboración entusiasta $y^{\prime}$ eficiente que en todo momento prestó la Junta de Aclelanto de Arica. A una hospitalidad generosa se agregó un esfuerzo decidido por asegurar que la organización del torneo fuera impecable. Arica demostró asi una vez más que posee amplios méritos para transformarse en el gran centro de encuentros intelectuales en la costa del Pacífico Sur.

Una vez finalizados los trabajos académicos del Seminario, un grupo cle participantes resolvió continuar reuniéndose informalmente para 
examinar la posibiliclad de adoptar actitudes más conducentes a la renlización de algunas metas integracionistas. EI Instituto de Estudios Internacionales no participó en estas deliberaciones puesto que su carácter estrictamente académico le impide adoptar una posición oficial frente a iniciativas políticas de cualquier especie. Estas reuniones informales resultaron en la creación del Grupo de Arica sobre cuyas deliberaciones informa más adelante el Sr. Antonio García.

Asimismo se ha incluido en este informe sobre el Seminario de Arica, la parte medular de una nota publicada por M. Jacques Vernant en el primer número para 1968 de Politique Etrangère, por reflejar un punto de vista evidentemente diferente y utilisimo para colocar el torneo en una perspectiva adecuada.

Craudio VéLIZ

\section{El diálogo latinoamericano en el Seminario de Arica}

El Seminario de Arica constituyó un interesante esfuerzo de coexistencia de dos tipos de diálogo: uno efectuado entre europeos sobre el tema cle la unificación y de la Comunidad Económica Europea, y otro entre latinoamericanos sobre la problemática de la integración en América latina. Para una justa comprensión de la naturaleza y nivel de estos dos diálogos coexistentes, debe decirse que los europeos se orientaron hacia la revisión de experiencias y los latinoamericanos hacia la definición de criterios teóricos y el examen global de problemas. Obviamente, la contribución europea se caracterizó por su açentuado pragmatismo, así como la contribución latinoamericana por la exigencia de perspectivas teóricas. Sin una estricta evaluación de estos dos ángulos de enfoque, no podría comprenderse, críticamente, la diversidad de las contribuciones europeas y latinoamericanas en el Seminario de Arica y la diversidád de los lenguajes empleados en los dos diálogos.

Dentro de este marco, debe señalarse que el Seminario de Arica no dispuso de verdaderas contribuciones acerca de algunos grandes temas, como los relacionados con la crisis de las instituciones de integración latinoamericana, la estrategia política de la integración y. la posición de los Estados Unidos frente a la integración latinoamericana. Tanto la problemática de las instituciones de integración como la referente a la estrategia política de la integración, fue abordada, por los ensayistas, en un plano de análisis formal y no como procesos 
dinámicos y estrechamente relacionados con los cambios estructurales en América latina.

Uno de los hechos más trascendentales del Seminario de Arica consistió en que en él se confrontaron tres grandes líneas de pensamiento, en relación con la problemática de la integración latinoamericana:

a) una que asigna la prioridad absoluta a la integración regional, enfocando la "integración nacional" como un proceso secundario e inducido;

b) una segunda que atribuye la prioridad absoluta a la integración nacional, desestimando radicalmente la problemática y el papel de la integración latinoamericana;

c) y una tercera que se afirma en dos puntọ de visța: el de que América latina no es libre de integrarse o no, debiendo escoger entre "integrarse clesde adentro y desde abajo" o ser "integrada desde arriba y desde afuera"; y el de que la realidad histórica impone la necesidad de afrontar, simultáneamente, los problemas de la integración nacional y de la integración latinoamericana:

El primer esquema fue enunciado por Lagos y Figueroa; el segundo por Frigerio y el tercero por Sunkel y Kaplan.

Dentro de este marco, Schatan, Kaplan y Figueroa expusieron un esquema de "integraciones escalonadas" y al nivel subregional.

Ėl análisis de la "problemática de la integración" reveló una serie de hechos fundamentales no sólo en relación a los procesos que vive la Ámérica latina, sino a las actitudes de su intelligentsia científicosocial. Esos hechos funclamentales son la materia de este juicio de perspectiva.

La primera serie de hechos podría constituir un diagnóstico de la próblemática de la integración:

a) Primero. América latina no es libre de escoger si se integra o no, ya que existe un proceso de integración liderizado por la gran. potencia hegemónica y por los grupos tradicionalmente dominantes en la sociedad latinoamericana: en consecuencia, es creciente el riesgo de que esa integración regional se efectúe desde afuera y desde arriba, por medio de limitadas políticas de desgravación y liberalización del comercio y ácentuando los términos de la dependencia externa. Es falso por lo tanto el dilema integración'/no integración.

b) Segundo. No existe, estratégicamente, un esquema de integración latinoamericana, sino dos: uno desde afuera y desde arriba y otro "desde adentro y desde abajo". Este segundo esquema corresponde a las fuerzas sociales identificadas en un propósito de cambio estructural y orientadas hacia la conquista de dos grandes objetivos estratégicos: el de armar a la América latina de una "facultad real de 
decisión" y de una verdadera "capacidad de negociación al nivel mundial"; y el cie asegurar el que los beneficios de la integración sirvan para transformar las miserables condiciones de vida de los pueblos latinoamericanos y para definir su nueva imagen de vida en el mundo;

c) Tercero. El esquema de "integración clescie adentro y desda abajo" ha sido expresado como línea de aspiraciones -definiéndose desde donde se inlegra y para quien se integra- pero no ha sido aún precisado como teoría y estrategia de cambio. En eso consiste uno de los más grandes desafíos a la intelligentsia cientílico-social de la América latina;

d) Cuarto. A esta altura de la historia hemisférica, no puede realizarse aisladamente una integración nacional prescindiendo de estructuras o formas políticoreconómicas de integración latinoamericana regional o subregional, ni puecle efectuarse una integración latinoamericana que no se fundamente y articule sobre procesos de enérgica integración nacional.

Cualquier esquema de integración desde adentro y desde abajo presupone, entonces, la adopción de una filosofía de simultancidad e indivisibilidad en los procesos de integración, al nivel nacional y latinoamericano. Dentro de este marco de la simultaneidad, deben precisarse y alinarse los nuevos enfoques teóricos clel problema, así como los posibles comportamientos estratégicos, en el orden económico financiero, cultural y político. Desde este punto de vista, en el Seminario se definieron dos líneas de aspiracionesi la de sustitución progiesiva del "esquema interamericano" por una esquema "exclusivamente latinoamericano", y la del reemplazo del "bilateralismo" por el "multinacionalismo", en las relaciones con las grandes potencias. Dentro de este orden cle ideas, se expuso la línea estratégica de "negociación latinoamericana" o multinacional de los programas internacionales de cooperación técnica y financiera.

El segundo núcleo de hechos podría relacionarse con la naturaleza del esquema tradicional de integración y con la estructura de la dependencia externa. Ese esquema tradicional de integración podría caracterizarse por una serie de elementos básicos:

a) La estructura de dependencia externa 'se fundamenta precisamente, en la integración de cada uno de los países latinoamericanos a la economia de la nación metropolitana '(España, Portugal, Gran Bretãna o Estados Unidos, en las diversas etapas históricas) ;

b) Ese tipo de integración lleva consigo, necesariamente, la incomunicación cntre las economías satelizadas, en el ámbito regional o 
subregional, como fenómeno consustancial a esos tipos coloniales de dependencia;

c) Ese status de incomunicación al nivel de los países latinoamericanos se expresa en la carencia de identificación de esos países en los objetivos estratégicos de la integración y el desarrollo, esto es, iden'tificación en el qué se persigue y para qué (Esquema Osvaldo Sunkel sobre la dependencia externa);

d) Es característico del esquema tradicional de integración y desarrollo, la carencia de finalidad popular $y$, consecuentemente, la total desestimación de las estructuras de participación democrática de los pueblos latinoamericanos;

e) Esa estructura de dependencia externa no sólo explica la incomunicación interior entre países latinoamericanos, sino también el "enfoque ideológico" y sin perspectiva pluralista de los grupos dominantes en esos países, en relación con los sectores no capitalistas del mercado mundial.

Dentro de este marco de examen de la problemática latinoamericana (Sunkel, Jaguaribe, Véliz, Sábato, Herrera, Schatan, Salgado, Lagos, Frigerio, Kaplan, Bianchi; Di Tella), debe señalarse la importancia que se asigná a los cambios estructurales como punto clave de cualquier nuevo esquema latinoamericano de integración y desarrollo (triángulo integración/desarrollo/autonomía, enunciado por Helio Jaguaribe). ¿Qué cambios estructurales y promovidos por quién? En el esquema tradicional, también se ha adoptado formalmente el principio de los cambios estructurales - como ha ocurrido en las diversas Cartas de Punta del Este- pero asumiendo que esos cambios pueden y deben ser promovidos por las clases dominantes en América latina y conducidos de acuerdo con los intereses de la potencia hegemónica y con sus propias aspiraciones de dominación social. El profundo desajuste entre la actual problemática nacional y regional de la América latina y el sistema político-social existente, se ha expresado en la crisis de ese sistema y, desde luego, de las instituciones regionales o "interamericanas" de integración.

Un tercer grupo de "hechos fundamentales" se relaciona con las posibilidades de aplicación inmediata de un esquema de integración "desde adentro y desde abajo", mediante un proceso de "integraciones escalonadas" y al nivel subregional. Este fue uno de los "puntos de confluencia" de las dos líneas de pensamiento expresadas con mayor claridad en el Seminario de Arica (ĺa orientada con un sentido de teorización de la experiencia histórica de la América latina y la conformada por un sentido absolutamente pragmá- 
tico) : la operabilidad inmediata de un esquema de integraciones escalonadas y progresivas, partiendo de formas de "asociación subregional" (Corporación Andina de Fomento, Marina Mercante Gran. Colombiana, etc.), o de "Comunidades Vecinales" (comunidada amazónica, comunidad del Plata, comunidad altiplánica del Lago Titicaca, etc.). La línea de mayor envergaclura teórica estuvo expresada por Sunkel, Jaguaribe, Schatan y Kaplan y la cle carácter pragmático por Lagos, Figueroa y Tomassini. El trasfondo de estal actitud coincidentc era la consicleración de que los procesos históricos no se conforman como alternativas racionales cle toclo o nada.

Lat más importante consecuencia de esta postura era, evidentemente, la posibilidad de que las nuevas fuerzas sociales de cambio en América latina pudiesen entrar a operar de inmediato, existienclo un clefiniclo objeto estratégico y una élite intelectual capar de inspimarlas $y^{\prime}$ expresarlas. El objetivo estratégico podría señalarse dentro clel marco de las integraciones escalonaclas, a nivel regional o subregional y en las cliversas esferas cle la rida latinoamericana (no sólo econónicass sino sociales, cultumles y políticas). Lil posibiliclad de negociar, multinacionalmente, programas de cooperación técnica y financiera con los Estaclos Uniclos o con otras grandes potencias o con orgalnizaciones de carácter mundial, por ejemplo, supondría la creación de estructuras políticas supranacionales de negociación y administración de esos programas, así como la capacidad de compartir - a nivel subregional- los complejos recursos culturales del mundo contemporáneo.

Dentro de este cuadro de factores, era interesante intentar una definición de la responsabilidad y el papel de la intclligentsia científicosocial, bien sea en la configuración de un nuevo esquema teórico de integración nacional y latinoamericana o en la orientación y promoción cle los modernos procesos de cambio estructuril en Améri(al latina.

La experiencia latinoamericana demuestra que sectores importantes de la intclligentsia han desempeñaclo, en cliversas coyunturas históricas, el papel de núcleos integradores de las fuerzas sociales identificadas con un propósito revolucionario de cambio. En las revoluciones de México, Bolivia y Cuba, la intelligentsia pudo desempeñar esta función de rúcleo integrador de los movimientos campesinos primero y posteriormente, de la movilización nacional de fuerzas populares iclentificaclas con la aspiración a un cambio estructural. Otro tanto ocurrió en el grupo de países - particularmente los australes- en los que el proceso de reformas tuvo un Liderazgo de clase media y partidos de orientación populista. Lo esencial de esta experiencia consiste 
en el papel histórico de la.intelligentsia, dentro de la línea de las reformas populistas o dentro del esquema latinoamericano de revolución nacional.

¿Qué es lo que podría proponerse como tarea inmediata para ese sector de la intelligentsia vinculado a las ciencias sociales y presumiblemente interesado en aclelantar los propósitos esbozadoș más arriba?

La respuesta clada por la mayoría de los latinoamericanos asistentes al Seminario de Arica, era ésta: primero, identificar a una élite cientílico-social con los objetivos, con las estructuras y con los medios de la integración descle y para la América latina, al nivel nacional y regional, y segundo, provocar una movilización de la intelligentsia latinoamericana en las múltiples clirecciones del cambio estructural.

La segunda preguntá era clè carácter operacional: ¿cómo identificar a esa élite de cientistas sociales de la América latina? La respuesta sc dio en diversos niveles:

a) diseñando un esquema latinoamericano de integración y desarrollo, y

b) utilizando plenamente los.instrumentos que actualmente existen y a los que están vinculados importantes sectores de la intelligentsia científico-social cle la América latina (agencias multinacionales de las Naciones Unidas como CEPAL, ILPES, CELADE, ICIRA u organismos universitarios del estilo del Instituto de Estudios Internacionales de li Universidad de Chile).

Sobre estas bases se definió una linea de acuerdo y compromiso, expresada en la constitución informal del Grupo de Arica y orientada en dos direcciones: hacia la integración de conocimientos científicos y sociales y hacia la acción inmediata. Las líneas de conocimiento (investigación, interpretación valorativa, divulgación) se desarrollarían por medio de organismos existentes o por crearse de inmediato, los que actuarían como "núcleos" nacionales, subregionales o regionales, de aproximación e integración paulatina de sectores de la intelligentsia latinoamericana. Estas líneas podrían lograr su máximo desarrollo en los grandes centros latinoamericanos de condensación de inteligencia científico-social (por medio de instituciones nacionales o multinacionales) como ocurre en Chile, México y Brasil.

Las "líneas de acción" podrían desarrollarse en varios sentidos:

a) el de proyección de métodos. y estructuras de "integración escalonada" y al nivel subregional (Comunidad Gran Colombiana, Comu- 
nidad Andina, Comunidad Amazónica o Comunidad Rioplatense, etc.) ;

b) el de proyección de órganos supranacionales de alcance politico, que tendería a eliminar, progresivamente, las formas coloniales. del bilateralismo y de la dependencia externa de tipo tradicional, estableciendo un sistema unificado de relaciones de la América latina con los Estados Unidos, con la Comunidad Económica Europea, con el sector socialista y comunista del mundo y con el proletariado de países atrasados de Asia y Africa, y

c) el de plena y sistemática utilización cle los instrumentos institucionales existentes, con un sentido de acción solidaria y unificada de los países latinoamericanos (en todo o en parte), ante los organismos multinacionales de carácter regional o mundial (Banco Mundial de Reconstrucción y Fomento, Banco Interamericano de Desarrollo, Comunidad Económica Europea, etc.) .

Dentro de estas grandes líneas, el Grupo de Arica definió su pensalmiento, su responsabilidad y su compromiso.

Antonio Garcis

\section{El Seminario de Arica visto desde Europa}

De las discusiones del Seminario de Arica se desprendieron, a mi juicio, algunas conclusiones:

1) El debate entre latinoamericanos se desarrolló a un nivel que permaneció demasiado teórico y abstracto. Se buscaron "modelos" y se discutieron "estrategias". Raramente se llegó a examinar situaciones concretas y a estudiar soluciones precisas nacionales, multinacionales o integradas.

2) La unificaçión europea ha sido el resultado de circunstancias históricas y de una estructura económica particulares. Históricamente la unificación de Europa bajo sus diversas formas, ha sido una adaptación a necesidades circunstanciales características de la postguermi: necesiclad de una unificación europea para la clistribución cle la ayuda norteameircana; para la reconstrucción económica y política de Europa frente a la "amenaza oriental"; necesidad de una unión europea esencialmente franco-alemana para materializar la antigua solidiaridad 
entre el Ruhr y la Lorena y para controlar el rearmamento alemán que se hizo necesario para contrarrestar la amenaza soviética.

Nada comparable existe en el mismo grado en América latina: Desde luego no existe la presión histórica: se podría encontrar dos motivos para la integración, pero actúan en sentidos contradictorios: por una parte, la amenaza "castrista", por otra, la "hegemonía norteamericana". De esta contradicción, nace la confusión en cuanto a la finalidad política de la integración.

Tampoco parece haber solidaridad económica transnacional (sino en ciertas regiones cuya importancia es limitada). Muy al contrario, 10 los polos de desarrollo en América latina están arraigados en el espacio nacional (por ejemplo, región de São Paulo). La repercusión de esos polos en cuanto a su desarrollo se eféctúa esencialmente al interior de este espacio. $2^{\circ}$ Los intercambios de países latinoamericanos están orientados hacia el exterior (Europa y América clel Norte); mientras que los intercambios intracontinentales siguen sienclo extremadamente limitados.

La estructura económica y soçial latinoamericana está caracterizada por la juxtaposición de un sector desarrollado y de un sector subdesarrollaclo, sin que haya propiamente movilidad entre los dos sectores.

A la inversa, los "Seis" constituian, hace mucho tiempo, un conjunto económicamente solidario; los intercambios internos eran traclicionalmente importantes aun antes de la creación de instituciones que facilitarian el desarrollo; tomanclo además en cuenta diferencias no clespreciables de nivel (Mezzogiorno italiano), una relativa homogeneiclad de desarrollo que preexistía entre los "Seis" y en el interior cle cada uno de ellos. Una vez que estas diferencias hayan sido claramente percibidas, me parece posible hacer dos observaciones:

a) Se debe aquí todavía, y más que nunca, deshacerse del nacionalismo y raciocinar sobre cosas y no sobre palabras; es inclispensable definir en qué consiste concretamente la "integración": cooperación bilateral o multilateral sobre proyectos limitados geográficamente y funcionalmente (financiamiento común de ciertos programas de interés multinacional en transportes o telecomunicaciones); acuerdos aduaneros, también uniones aduaneras entre dos o varios paises; creación de organismos comunitarios de competencia limitada que tengan un papel consultativo o poderes de aclministración. Todos estos esquemas son tal vez aplicables.

b) Se trata también de contestar a la pregunta: ¿Por qué?, o más precisamente, ¿para quién se va a integrar? Integrar para desarrollar, sin clucla, pero ¿qué tipo de desarrollo se escoge? ¿Con cuál perspectiva 
y en el interés de quién? Estas preguntas, como se puecle prever, fueron hechas por la mayoría de los participantes latinoamericanos en el Seminario de Arica.

En cuanto a la organización del Seminario, fue perfecta: la Unirersiclad cle Chile y el gobierno chileno actuaron en la mejor forma posible, El Sr. Clauclio Véliz y el Sr. Osvalclo Sunkel, quienes dirigieron la organización de los trabajos y de los paseos, pueden felicitarse cle un éxito al cual aplaudimos.

JACQUES VERNANT 